http://doi.org/10.15359/ree.13-2.9

\title{
La población estudiantil en el sétimo año de la educación pública costarricense
}

\author{
Magdalena Alfaro Rodríguez \\ Académica de la División de Educología del CIDE* de la Universidad Nacional \\ Heredia, Costa Rica
}

Alejandra Gamboa Jiménez ${ }^{2}$

Académica de la División de Educación para el Trabajo del CIDE* de la Universidad Nacional

Heredia, Costa Rica

Susana Jiménez Sánchez ${ }^{3}$

Heredia, Costa Rica

Jorge R. Martín Pérez ${ }^{4}$

Académico de la División de Educación para el Trabajo del CIDE* de la Universidad Nacional

Heredia, Costa Rica

Andrea Ramírez González ${ }^{5}$

Académica de la División de Educación Rural del CIDE* de la Universidad Nacional

Heredia, Costa Rica

Marie-Claire Vargas Dengo ${ }^{6}$

Académica de la División de Educación Básica del CIDE* de la Universidad Nacional

Heredia, Costa Rica

* Centro de Investigación y Docencia en Educación de la Universidad Nacional

${ }^{1}$ Magíster en Educación con énfasis en Docencia Universitaria de la Universidad Nacional. Licenciada en música con énfasis en enseñanza musical. Actualmente, es académica de la División de Educología del CIDE. Es integrante del cuarto equipo del proyecto Perfiles, dinámicas y desafíos de la educación costarricense.

Correo electrónico: magdar613@hotmail.com

²- Máster en Psicopedagogía de la Universidad de La Salle, con estudios en Ciencias de la Educación con énfasis en Orientación de la Universidad Nacional. Actualmente, es la subdirectora de la División de Educación para el Trabajo del Centro de Investigación y Docencia en Educación Participó en el tercer equipo del proyecto Perfiles, dinámicas y desafíos de la educación costarricense.

Correo electrónico: Igamboa@una.ac.cr

${ }^{3}$ Máster en Tecnología Educativa de la Universidad Estatal a Distancia. Licenciada en Enseñanza de la Química de la Universidad de Costa Rica y en Ciencias de la Educación con énfasis en Docencia de la Universidad Estatal a Distancia. Con estudios en educación primaria. Actualmente, es académica de la División de Educología del CIDE. Coordinó el tercer equipo del proyecto Perfiles, dinámicas y desafíos de la educación costarricense.

Correo electrónico: sujisa@gmail.com

${ }^{4}$ Magíster en Educación con énfasis en Docencia Universitaria de la Universidad Nacional, Licenciado en Economía y Licenciado en Cibernética Matemática de la Universidad de la Habana. Actualmente, es académico de la División de Educación para el Trabajo del CIDE. Coordina el cuarto equipo del proyecto Perfiles, dinámicas y desafíos de la educación costarricense.

Correo electrónico: jorgeramonmartin@yahoo.es

${ }^{5}$ Licenciada en Enseñanza de la Matemática de la Universidad de Costa Rica y con estudios en Educación Primaria. Actualmente, es académica de la División de Educación Rural del CIDE. Fue integrante del tercer equipo del proyecto Perfiles, dinámicas y desafíos de la educación costarricense.

Correo electrónico: aramgo@costarricense.cr

${ }^{6}$ Máster en Currículum e Instrucción de The George Washington University, USA. Actualmente, es académica de la División de Educación Básica del CIDE. Es integrante del cuarto equipo del Proyecto perfiles, dinámicas y desafíos de la educación costarricense.

Correo electrónico: marie d claire@yahoo.com 
Recibido 31 de julio de 2009 • Aceptado 14 de setiembre de 2009

Resumen. El estudiantado de sétimo año comparte características que son analizadas en este trabajo, en función del adecuado desempeño docente en la mediación de su aprendizaje, a partir de los resultados de la investigación realizada para el diseño del perfil de desempeño de las y los docentes de este nivel educativo (Alfaro et al., 2008a).

Se concluye que el desarrollo biopsicosocial y el estadio maduracional del tránsito niñez-preadolescencia-adolescencia, debe ser del dominio del profesorado de este nivel, así como el contexto socioeconómico familiar y social de sus estudiantes.

Palabras clave. Estudiantado, permanencia, promoción, sétimo año, educación secundaria, perfil docente.

Abstract. Seventh grade students share personal characteristics that are analyzed in this paper based on the teachers' performance of the proper mediation for the students' learning. The results of an investigation done with the model of teachers' profiles at this level will be the basis for this paper (Alfaro et al, 2008a).

We conclude that the biopsychosocial development and maturational stage of the transit-preteen-teen children must be the domain of faculty at this level, as well as, the socioeconomic context of family and social contexts of their students.

Key words. Students, attendance, promotion, seventh grade, secondary level, teachers profile.

\section{Introducción}

En el año 2007, en el Centro de Investigación y Docencia en Educación (CIDE) de la Universidad Nacional de Costa Rica, específicamente, en el marco del proyecto Perfiles, Dinámicas y Desafíos de la Educación Costarricense, se gestó un proceso de investigación con el fin de construir el perfil de desempeño de los y las docentes de sétimo año. Como resultado de este proceso, se identificaron aquellos atributos, características, cualidades y condiciones del desempeño que son factores claves para propiciar una relación interpersonal positiva docentes-estudiantes y que contribuyen a la transición de la escuela al colegio, así como a la permanencia, el mejoramiento de la promoción y el éxito de los y las estudiantes de sétimo año dentro del sistema educativo formal (Alfaro et al., 2008a).

Tal como lo establece Rojas (2007), en el documento Hacia un modelo educativo para elevar la calidad de la educación costarricense elaborado por CONARE, todo docente debe "conocer sobre el desarrollo integral de los educandos del nivel que tendrá a su cargo para adecuar las acciones educativas con el fin de contribuir a la formación para el desarrollo integral del educando (cognitivo, afectivo, psicomotor), para desarrollar al máximo sus potencialidades" (p. 10). Esta afirmación lleva a pensar que todo docente que tenga a su cargo sétimo año debe estar al tanto de todas las particularidades que esta población estudiantil tiene. Cabe, entonces, preguntarse: ¿qué características intelectuales, sociales, físicas, morales, emocionales y psicológicas tiene el estudiantado de sétimo año? ¿Qué los hace permanecer en el colegio? ¿Cómo los conciben sus docentes? En este artículo, se resumen las respuestas que, la recolección y el análisis de los datos de la investigación realizada, da a estas interrogantes. 


\section{Perspectiva teórica}

\section{El estudiantado en sétimo año: características de la preadolescencia y la adolescencia}

Existen rasgos propios que caracterizan al estudiantado en las etapas de la preadolescencia y la adolescencia; al respecto, podría decirse que cuando se es preadolescente existen, aún, rasgos de la etapa de la niñez y, ya, empiezan a vislumbrarse rasgos propios de la adolescencia.

Se ha dicho, teóricamente, que una de las características más predominantes en ambas etapas, son la manifestación de crisis propias del desarrollo, las crisis aparecen cuando surgen cambios y éstos producen o profundizan nuevas necesidades, que la Asociación Nacional de la Escuela Media de los Estados Unidos (1995) (citado por Castro et al., 2007), puntualiza como:

1. Intelectuales. Los estudiantes jóvenes son curiosos, están motivados al logro cuando son retados y son capaces de resolver problemas y pensar de manera compleja.

2. Sociales. Existe una intensa necesidad de pertenecer y ser aceptado por sus compañeros en la búsqueda de su lugar en el mundo. Se hallan involucrados en formar y cuestionar sus identidades en muchos niveles.

3. Físicas. Ellos maduran a diferentes ritmos y experimentan un crecimiento rápido e irregular, con cambios que causan movimientos extraños y descoordinados.

4. Emocionales y psicológicas. Son vulnerables, autoconscientes y, a menudo, experimentan cambios impredecibles de temperamento.

5. Morales. Son idealistas y quieren tener impacto en hacer del mundo un mejor lugar.

Tanto en la preadolescencia como en la adolescencia, ocurren transformaciones profundas en todo nivel, que producen confusión y desorientación, no hay comprensión de sí mismo, ni del entorno, lo que genera gran insatisfacción, descontento, ansiedad; se afecta la comunicación entre generaciones y se manifiesta cierta desconfianza y, hasta, desacato hacia la autoridad. Por lo general, en esta etapa, no se permite la intromisión de las personas adultas, se crea y se vive en una especie de mundo propio clandestino, conocido y accesible sólo en círculos de contemporáneos muy allegados y selectos; se incrementa el deseo de autonomía con sentido de soledad.

Las tareas y demás obligaciones escolares, unidas a las exigencias paternas y a las demandas de la convivencia en el grupo de iguales, son de gran relevancia en la construcción de la identidad personal.

En la etapa de la adolescencia surge una gran admiración y construcción de ídolos o modelos por seguir, con los que esta población se identifica plenamente, ya sea por la música, por el físico, por el léxico o por predilecciones determinadas; la industria de los medios de comunicación promueve nuevas y diversas necesidades en este sentido.

La Tabla 1 presenta algunas de las principales transformaciones que se producen en la preadolecencia e inicios de la adolescencia, ordenadas según seis aspectos: físicos, sociales, emocionales o psicológicos, intelectuales, educacionales y morales. 
Tabla 1

Principales transformaciones en el estudiantado de sétimo año del sistema educativo costarricense: características por áreas del desarrollo integral

\begin{tabular}{|c|c|c|}
\hline Físicos & Sociales & Emocionales o psicológicos \\
\hline $\begin{array}{l}\text { - Cambios hormonales: } \\
\text { Hombres: por lo general, los } \\
\text { cambios se inician a partir de los } \\
14 \text { años, gran cantidad de testos- } \\
\text { terona: crecimiento de órganos } \\
\text { genitales, laringe, vello púbico, } \\
\text { vello en el rostro y axilas, pro- } \\
\text { ducción de esperma, emisiones } \\
\text { nocturnas. } \\
\text { Mujeres: por lo general, los cam- } \\
\text { bios se inician a los } 12 \text { años, cre- } \\
\text { cimiento de órganos internos y } \\
\text { externos, producción de hormo- } \\
\text { nas (estrógenos y progesterona), } \\
\text { ensanchamiento de caderas y } \\
\text { crecimiento de pechos, produc- } \\
\text { ción de óvulos. } \\
\text { Estatura y peso. }\end{array}$ & 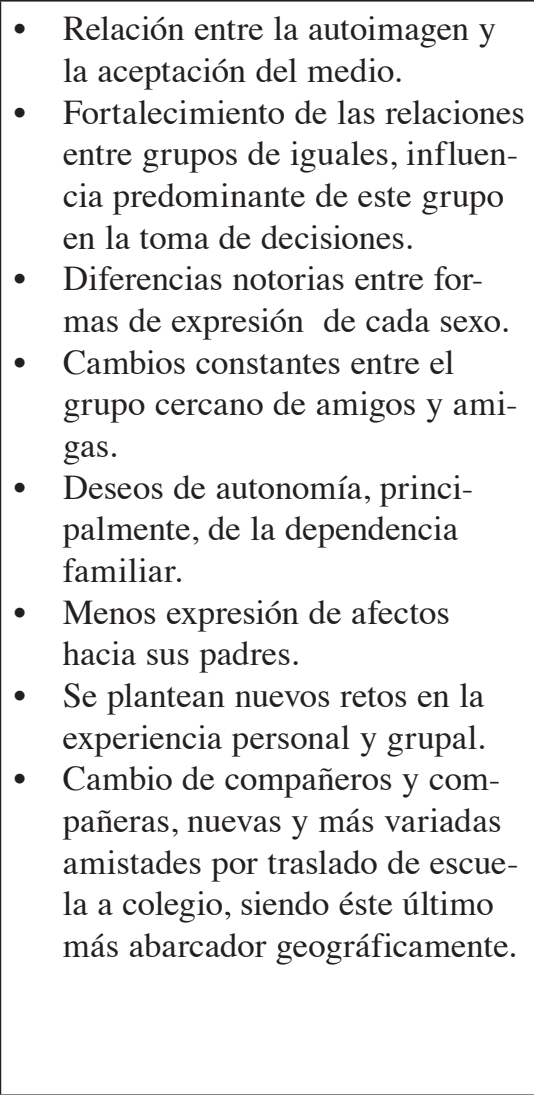 & $\begin{array}{l}\text { - Cambios de autoimagen. } \\
\text { - Cambios en autoconcepto, de ser } \\
\text { los mayores de la escuela, a los } \\
\text { menores en el colegio. } \\
\text { - } \text { Intereses por sexualidad, en } \\
\text { especial por sexo opuesto, más } \\
\text { desarrollado en las mujeres. } \\
\text { - Timidez, vergüenza, deseos de } \\
\text { - } \text { ser aceptados. } \\
\text { - } \text { Altasqueda de identidad. } \\
\text { - } \text { Cer más atractivos o atractivas. } \\
\text { - Sensibilización por problemas de } \\
\text { - } \text { sus iguales. } \\
\text { Expresiones infantiles, espe- } \\
\text { cialmente, cuando se tiene poca } \\
\text { seguridad ante un evento. } \\
\text { - Sensibilidad, emotividad por lo } \\
\text { actual, sin proyección a futuro. } \\
\text { - Reconocimiento de nuevas habi- } \\
\text { lidades. } \\
\text { Inquietudes por nuevas experien- } \\
\text { cias. } \\
\text { externos e internos. }\end{array}$ \\
\hline Intelectuales & Educacionales & Morales \\
\hline $\begin{array}{l}\text { - Nuevos retos y responsabilidades } \\
\text { académicas. } \\
\text { Menos dependencia de realizar } \\
\text { los trabajos con sus padres o res- } \\
\text { ponsables. } \\
\text { Transición del pensamiento con- } \\
\text { creto al formal, lo que le implica } \\
\text { relaciones y análisis más com- } \\
\text { plejos. Piaget. Esto se encuentra } \\
\text { ligado a la capacidad crítica en } \\
\text { la que algunos y algunas adoles- } \\
\text { centes comienzan a manifestar } \\
\text { en esta transición. }\end{array}$ & $\begin{array}{l}\text { - Transición de un ciclo a otro y } \\
\text { de primaria a secundaria. } \\
\text { Multiplicación de docentes y } \\
\text { más distantes. } \\
\text { - } \text { Aumento de materias y deberes. } \\
\text { Transformaciones evaluativas } \\
\text { (trabajo diario, extraclase y exá- } \\
\text { menes). } \\
\text { - Cambio de horarios y rutinas. } \\
\text { Independencia de familia para } \\
\text { tareas colegiales. } \\
\text { Diferente disciplina, le reclaman } \\
\text { más madurez. }\end{array}$ & 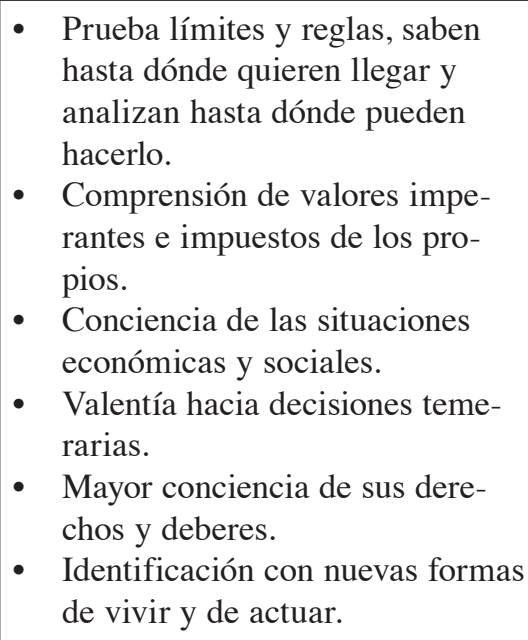 \\
\hline
\end{tabular}

Continúa... 


\begin{tabular}{|c|c|c|}
\hline Intelectuales & Educacionales & Morales \\
\hline $\begin{array}{l}\text { - El interés primordial hacia lo } \\
\text { académico es relevante cuando } \\
\text { ayuda en su presente. }\end{array}$ & 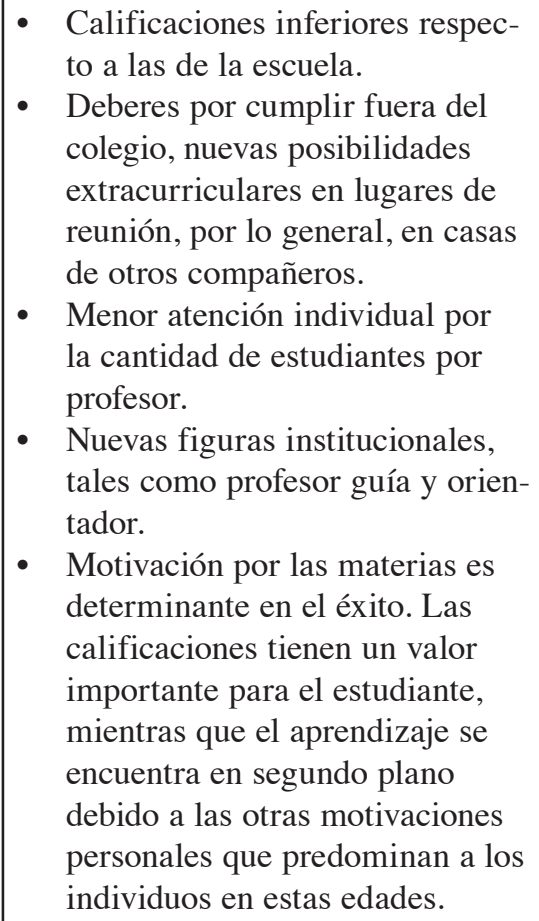 & $\begin{array}{l}\text { - Capacidad de riesgo y expresión } \\
\text { de los propios ideales. } \\
\text { Existen mitos alrededor de la } \\
\text { persona en esta edad, tales como } \\
\text { obsesión por el sexo, rebeldía, } \\
\text { indecisión, malhumorada, entre } \\
\text { otros. }\end{array}$ \\
\hline
\end{tabular}

Nota: Tomado de Alfaro et al. (2008).

\section{El aprendizaje de los estudiantes de sétimo año}

Existen diversas teorías que explican sobre el proceso de aprendizaje de las personas y de cómo se produce. Dentro de las teorías que se han generado respecto al desarrollo del pensamiento, se reconoce la obra del psicólogo ruso Vigotsky (1896-1934) quien presentó sus aportes en la segunda década del pasado siglo, y argumentó el aprendizaje como una actividad social que se da en un contexto inmediato e histórico en el que el individuo está estrechamente relacionado con el ambiente, la zona de desarrollo próximo, el docente, cuyo rol fundamental es el de mediar el proceso de aprendizaje.

La teoría de Vigotsky se basa en que el habla tiene un origen social y que el lenguaje sigue al pensamiento racional que influye en su naturaleza, de ahí que el lenguaje es fundamental para cualquier conocimiento, surge por la necesidad de comunicación entre las personas y se desarrolla en un contexto social. Asimismo, el desarrollo del lenguaje posibilita la entrada en la cultura, pues sus funciones mentales superiores, el lenguaje y el pensamiento se desarrollan, primero, en la interacción del ser humano con otros individuos. Esas funciones interpersonales suceden gradualmente intrapersonales, a medida que el ser humano sea consciente de su significación (Gastón, 1996).

Para Vigotsky, en el aprendizaje debe existir una mediación entre los conocimientos y el estudiante, por lo que parte de un enfoque histórico cultural para ubicarla dentro de un contexto escolar y explicarla en la propuesta de la Zona de Desarrollo Próximo, en la que se resalta la importancia de la interacción social dinámica en la construcción del conocimiento (Labarrere, 2008). El proceso de aprendizaje se apoya en los objetos, el medio ambiente y las situaciones que acompañan al ser humano en cada momento, de ahí que sea un proceso que incluye las relaciones entre los individuos y, por tanto, no sólo se da por la interacción del individuo y el medio cultural o social que lo rodea, 
sino que esta relación es esencial en el mismo proceso de aprendizaje que incluye al que aprende, al que enseña y la relación entre ambos.

Prensky (2001) señala que los actuales estudiantes, a lo largo de sus vidas, han estado rodeados por el uso de computadoras, juegos de vídeo, música digital, vídeos, teléfonos celulares y otros juguetes y herramientas propios de la era digital, por lo que sienten atracción por todo lo relacionado con las nuevas tecnologías (García, Portillo, Romo y Benito, 2007). Gracias a este ambiente ubicuo y al volumen de su interacción con la tecnología, el estudiantado nacido a partir de los años 80 piensa y procesa la información de manera diferente a sus antecesores.

De acuerdo con las ideas de Prensky (2001), podemos identificar, entonces, personas que nacieron y han crecido en la era digital, incluso, los distingue de los inmigrantes digitales. En la Tabla 2, se describen las características de ambos grupos. Para los inmigrantes digitales, la capacidad de abordar procesos paralelos de los nativos digitales no son más que comportamientos con apariencia caótica y aleatoria.

Tabla 2

Nativos frente a inmigrantes digitales

\begin{tabular}{|l|l|}
\hline \multicolumn{1}{|c|}{ Nativos digitales } & \multicolumn{1}{|c|}{ Inmigrantes digitales } \\
\hline Menores de 30 años. & Personas entre 35 y 55 años. \\
\hline $\begin{array}{l}\text { Tienen una habilidad innata en el lenguaje y } \\
\text { en el entorno digital. }\end{array}$ & $\begin{array}{l}\text { Se han adaptado a la tecnología y hablan su } \\
\text { idioma, pero con cierto acento. }\end{array}$ \\
\hline $\begin{array}{l}\text { Las herramientas tecnológicas ocupan un } \\
\text { lugar central en sus vidas y dependen de ellas } \\
\text { para todo tipo de cuestiones cotidianas, tales } \\
\text { como estudiar, relacionarse, comprar, infor- } \\
\text { marse o divertirse. }\end{array}$ & $\begin{array}{l}\text { Son fruto de un proceso de migración digi- } \\
\text { tal que supone un acercamiento hacia un } \\
\text { las Tecnologías de la Información y la } \\
\text { Comunicación (TIC). }\end{array}$ \\
\hline $\begin{array}{l}\text { Comparten y distribuyen información con } \\
\text { toda naturalidad. }\end{array}$ & $\begin{array}{l}\text { Tienden a guardar en secreto la información } \\
\text { (el conocimiento es poder). }\end{array}$ \\
\hline $\begin{array}{l}\text { Son capaces de tomar decisiones de una forma } \\
\text { rápida y en ambientes complejos. }\end{array}$ & $\begin{array}{l}\text { Los procesos de actuación de los inmigrantes } \\
\text { suelen ser reflexivos y, por tanto, más lentos. }\end{array}$ \\
\hline $\begin{array}{l}\text { Los juegos electrónicos que usan son más } \\
\text { complejos, implicando la participación y la } \\
\text { coordinación de más jugadores. Asimismo, } \\
\text { crean recursos: herramientas, armas, espacios, } \\
\text { universos propios. }\end{array}$ & $\begin{array}{l}\text { Los juegos electrónicos utilizados por ellos en } \\
\text { décadas anteriores eran lineales en funciona- } \\
\text { miento y objetivos. }\end{array}$ \\
\hline
\end{tabular}

Nota: Elaboración del equipo investigador, con base en García et al. (2007). 
Según García, et al., (2007) y Prensky (2001), otras características importantes de los nativos digitales es que:

1. Absorben, rápidamente, la información multimedia de imágenes y vídeos, igual o mejor que si fuera texto, prefiriendo los formatos gráficos a los textuales.

2. Consumen datos, al mismo tiempo, de múltiples fuentes, afrontando distintos canales de comunicación simultáneos.

3. Esperan respuestas instantáneas.

4. Permanecen comunicados permanentemente.

5. Crean, también, sus propios contenidos.

6. Son multitarea, es decir, hacen varias cosas al mismo tiempo.

7. Utilizan el acceso hipertextual en vez del lineal.

8. Funcionan mejor trabajando en red.

Los y las estudiantes nativos digitales están más predispuestos a utilizar las tecnologías en actividades de estudio y de aprendizaje que lo que los procesos educativos les pueden ofrecer. Por ello, se requiere que el profesorado, el cual, en su mayoría es migrante digital, reconsidere la metodología que utiliza y el contenido que se quiere que aprendan (Prensky, 2001).

\section{Consideraciones metodológicas de la investigación realizada}

Como se mencionó en la introducción, la investigación que dio origen a este artículo, se realizó en el proyecto de investigación del CIDE denominado Perfiles, dinámicas y desafíos de la educación costarricense, con el fin de abordar el tema de la Construcción del Perfil Docente para el Sétimo Año de la Educación Pública. Dicha investigación utilizó un enfoque metodológico mixto (Hernández, Fernández \& Baptista, 2004), llamado así por cuanto aborda el problema tanto desde un enfoque cualitativo como cuantitativo, aunque el enfoque predominante en esta investigación fue el cualitativo. La información se obtuvo mediante la realización de observaciones de aula, entrevista a docentes, directores, orientadores y estudiantes de sétimo año, complementadas con talleres con estudiantes y profesores, todo ello aplicado en nueve colegios de zonas urbanas y rurales de las provincias de San José, Guanacaste y Cartago.

La selección de los Centros educativos fue intencionada y se basó en que contaran con Proyectos institucionales en funcionamiento, enfocados a mejorar la retención y la promoción en el Sétimo año, ya que se buscaba la detección de elementos de éxito en el desempeño docente en diversos contextos, de ahí se deriva la diversidad de instituciones.

Se procuró incluir a la totalidad de docentes, orientadores y directores; y en cuanto al estudiantado, se tomaron de dos a cuatro grupos completos, que representaron más de la tercera parte de la población en ese nivel en cada centro. Todos los instrumentos utilizados (guía de observación, cuestionario, entrevista, guías para los talleres) fueron validados mediante: a) expertos en investigación educativa, pedagogía y currículum, con grados académicos de Maestría y Doctorado; b) pilotaje; es importante recalcar que se utilizó en cada instrumento, trabajo de grupo, talleres, cuestionarios y entrevistas, el criterio de confidencialidad de la información para la protección de las personas participantes.

Para la realización de este artículo se consideraron los resultados de las observaciones de aula y de los talleres con estudiantes y profesores, pues es en éstos en los que se reflejan las características de la población estudiantil de sétimo año. 


\section{Resultados y discusión}

\section{Observaciones}

Desde puestos de observación satelital y usando un guía de observación, el equipo de investigación aplicó la observación no participante. En relación con la caracterización de la población estudiantil, se enfocaron categorías de análisis, tales como las actitudes de esta población, las cuales tienen íntima relación en las dinámicas que se dan en el clima de aula, la cantidad de estudiantes que se vinculan en el proceso que se da en el aula y algunos datos que tienen explícita relación con la deserción intranual.

Las actitudes son concreciones de valores. Sirven para ordenar y dotar de significado a aspectos del medio social en los que la persona se mueve; es un ejemplo de actitud tener relaciones interpersonales equilibradas con los compañeros (Guitart, 2002). Las actitudes más sobresalientes y que presentan mayor frecuencia en las observaciones realizadas, se relacionan con ciertas actitudes, tales como que la población estudiantil habla constantemente entre iguales y que lo hacen al mismo tiempo, lo que produce que no se puedan escuchar entre ellos y ellas, se distraen de las tareas que el o la docente les dice que realicen; además, producen distractores para los estudiantes que se encuentran realizando alguna tarea de trabajo cotidiano, tales como tirarse bodoques de papel y basura.

Existen otras manifestaciones propias de los estudiantes como burlas hacia otros compañeros, ya sea por responder a una pregunta erróneamente, por no saberse las tablas, por no pronunciar bien una palabra. También, algunos estudiantes utilizan vocabulario soez y se ríen o burlan de algunos(as) profesores(as), ya sea por su forma de hablar, vestir o de relacionarse con el grupo.

Como parte natural en la dinámica del aula, surgen entretenimientos regulares en la población estudiantil, tales como enviarse papelitos, o mensajes por celular, todo esto a la vista del docente, $\mathrm{y}$, en otros casos, de forma clandestina para no recibir llamadas de atención, compartir alimentos, mascar chicle, tomar agua constantemente de sus botellas (este rasgo se observa especialmente en las mujeres), pasarse fotografías, peinarse, perfumarse y, la más frecuente: hablar sobre temas de su interés. De acuerdo con Obiols y Di Segni (1995), éstos son rasgos de los adolescentes postmodernos, para quienes la fugacidad, el culto al presente y el hedonismo son parte de su diario vivir, por lo que disfrutan de lo breve e intenso.

Llama la atención los recursos que utilizan algunos estudiantes para distraerse cuando tienen momentos libres, o porque escogen darse momentos de ocio dentro de las tareas escolares, entre ellos se encuentran los iPods, los discman y los celulares; éstas tecnologías son muy atractivas para ellos cuando la clase resulta ser magistral y cuando topan con un profesor que tiene un grado de permisividad; no obstante, los que no poseen este tipo de tecnología, recurren a las conversaciones con personas que les atraen. Puede observarse cómo el uso de tecnología, propio de los nativos digitales (Prensky, 2001) está presente en las aulas de sétimo año que participaron de la investigación.

Algunas de las manifestaciones que se dan, según sea el sexo, se aprecian en las relaciones interpersonales entre los hombres, con señales de trato poco afectivo, con evidencia de golpes, ofensas o tirándose cosas. En contraposición, están las señales que se evidencian en el grupo de mujeres, quienes evidencian las relaciones basadas en cariños, pasando la mano por el cabello de otra compañera, prestándose cosméticos y utensilios para embellecerse.

En estos resultados, surgen muestras de la diversidad de la población estudiantil de sétimo año, ante lo que se hace necesario que los y las docentes: a) tengan tolerancia y respeto a las diferencias individuales; b) se capaciten en la atención a la diversidad; c) detecten problemas, oportunamente, para mediar en la construcción de las soluciones; d) valoren las sugerencias planteadas por el alumno 
y e) consideren la percepción del alumnado como uno de sus medios más eficaces de aprendizaje; por lo que deben tener en cuenta sus estrategias y actuaciones en clase (Alfaro et al., 2009).

\section{Los talleres}

Son aquellas actividades que se organizaron con los sujetos de estudio, con el fin de obtener información complementaria a las demás técnicas aplicadas, tanto de la situación actual del sujeto investigado, como ideas para su perfeccionamiento. En el marco de esta investigación se realizaron talleres con estudiantes y con docentes de sétimo año, cada uno con sus respectivos objetivos y técnicas.

\section{Análisis del taller con estudiantes de sétimo año}

En las sesiones grupales con la población estudiantil, se trabajó con un instrumento llamado tarjetas activas. Éstas consisten en ejes o preguntas generadoras usadas para promover la reflexión, el análisis y la discusión alrededor de los temas de interés investigativo. Para este caso particular, se usaron dos grandes preguntas con sus subtemas, planteadas en primera persona como una estrategia para que el sujeto interiorice y responda la pregunta desde su vivencia personal:

1. ¿Qué me ayuda en mi rendimiento académico?

2. ¿Qué me motiva a permanecer en el colegio?

En relación con la primera pregunta, las respuestas de los estudiantes se muestran en la Figura 1. Para algunos(as) estudiantes es indispensable la relación que exista con los y las docentes, la cual debe ser una relación en la que brinden apoyo y buenos consejos; sin embargo, mucho(as) dicen que "algunos nos odian".

También es importante la ayuda de los y las docentes para aprobar las materias, ya sea con explicaciones claras que aclaren las dudas que se presentan, o con los trabajos extraclase; el brindar consejos hace que sientan apoyo por parte del profesorado, además de la motivación y el impulso que éstos brindan para seguir adelante.

Otras razones son: trabajar en clase, cumplir con los trabajos extraclase que les asignan, realizar las tareas y los materiales que utilizan como guía; además de la asistencia y el horario adecuado, la parte espiritual es muy importante para salir adelante y superarse. Otros aspectos que les ayuda en el colegio son estudiar con música, en voz alta, concentrarse, presentar los trabajos, además de reconocer el esfuerzo que hacen ellos mismos.

El papel de la familia es fundamental, como pilar de apoyo en los estudios, ya sea "ayudándonos a estudiar", "en los gastos", "los consejos", "nos impulsan y nos brindan cariño", "nos levantan y nos envían al colegio a estudiar, para que tengamos un futuro". Además "podemos ofrecer a nuestros padres los logros y que se sientan orgullosos del esfuerzo que ellos están realizando", pues es un beneficio, a futuro, tanto para ellas y ellos, como para sus padres y sus futuras familias. Dentro del marco de la familia, se evidencia que la madre es la que más mencionan los y las jóvenes como apoyo, consejera, quien les ayuda con los gastos, les levanta para ir al colegio y les brinda buenos ejemplos. Estas descripciones, con respecto a los padres, brindadas por los estudiantes, no coinciden con lo que Gavilán y D'Onofrio 


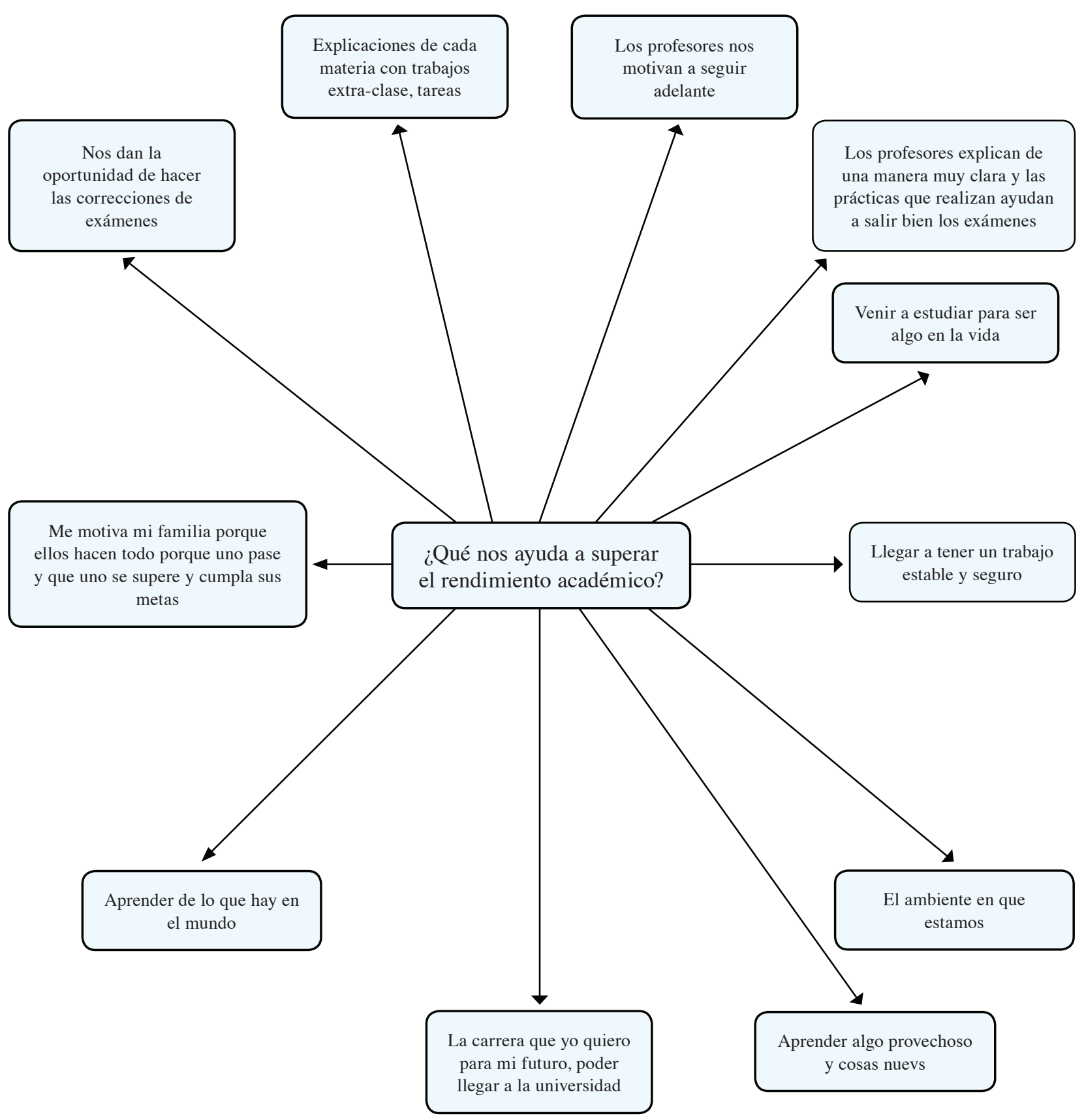

Figura 1. ¿Qué ayuda a los estudiantes de sétimo en su rendimiento?

Nota: Tomado de Alfaro et al. (2008a)

(2003) afirman "se ha eclipsado la autoridad paterna, que tiene un común denominador en su modo de ejercerse: miedo e inseguridad de los padres (...) estos estilos de padres no permiten al adolescente incorporar una imagen claramente diferenciada del adulto, separada de por sí por la madurez generacional" (p. 8). Podríamos pensar, entonces, que los padres y las madres de estos estudiantes asumen el rol de figuras que guían la formación de sus hijos e hijas, pero esto sería tema de otro trabajo. 
Con respecto a qué les motiva a permanecer en el colegio, se agruparon las respuestas de los estudiantes en doce categorías que se presentan en la Figura 2. Cabe resaltar que, para algunos o algunas estudiantes, un punto importante de motivación en la permanencia en el colegio son las ayudas que se brindan en la institución, como lo son las becas, las aulas educación especial y los clubes que se realizan.

En las clases, les ayuda con su rendimiento académico prestar atención, realizar dinámicas y aprender cosas nuevas de las materias.

Otras razones aportadas, en relación con la permanencia en el colegio, es su relación con el grupo de pares; se evidencia la tendencia de los jóvenes a asistir al colegio por la convivencia que experimentan con personas de su edad, compartir con sus amigos y amigas, conocer personas nuevas y aprender de ellas, divertirse, ir a ver muchachos guapos y mujeres bonitas. Las amistades del colegio son muy importantes para los jóvenes, ya que mencionan, en repetidas ocasiones, que lo que les ayuda es el apoyo de las amistades.

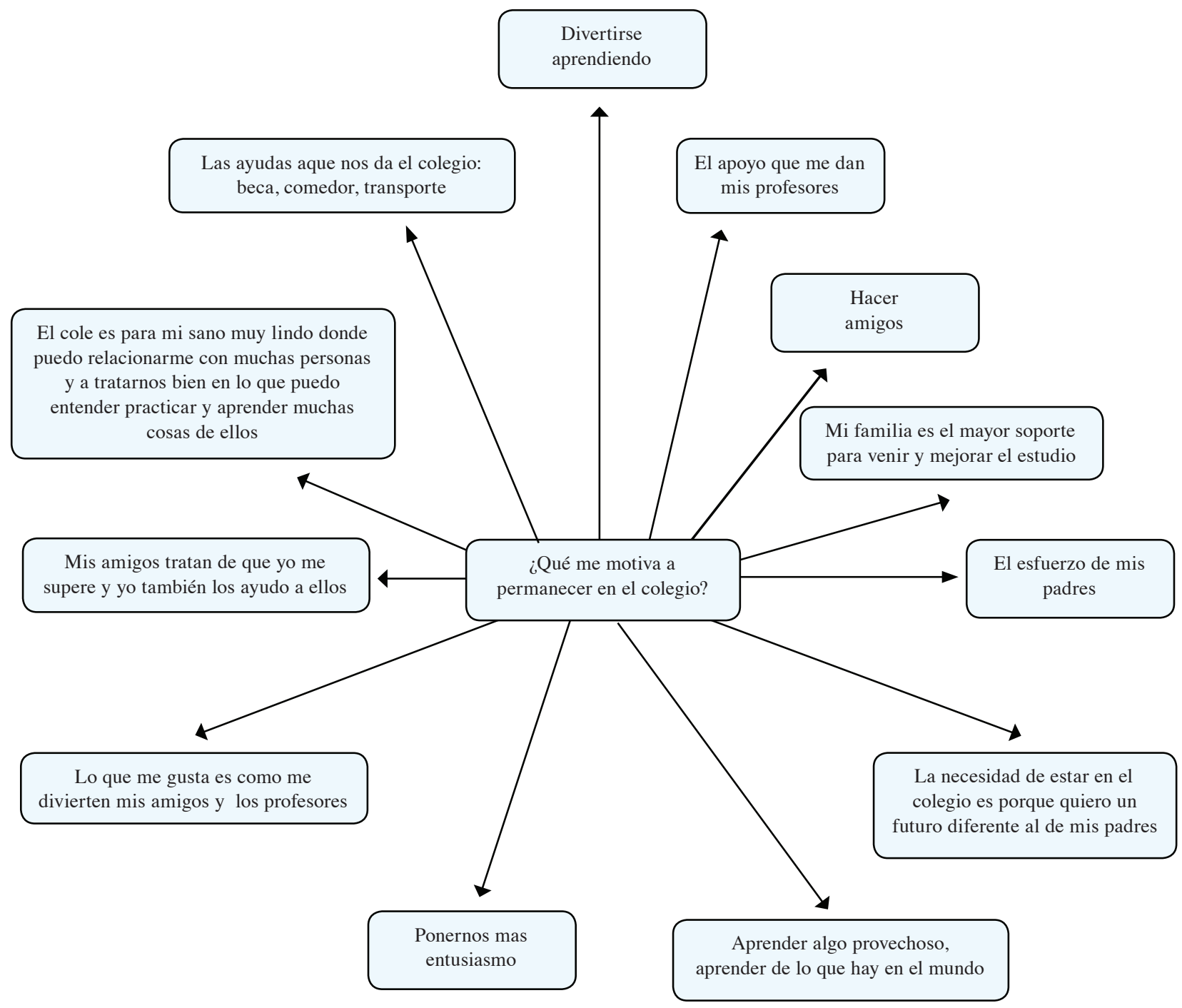

Figura 2. ¿Qué motiva a los estudiantes de sétimo a permanecer en el colegio?

Nota: Tomado de Alfaro et al. (2008a) 
Para los y las estudiantes, el apoyo de las personas de la comunidad es motivador para permanecer en el colegio, ya que les aconsejan que estudien, les impulsan y les ayudan a realizar los trabajos que les asignan. En relación con la comunidad, los ejemplos de las personas son valiosos: los positivos, por ejemplo, de algunas personas que son responsables en la comunidad, y los negativos, que son los que les inspiran a querer "ser alguien".

Se evidencia la importancia de pensar en el futuro, las metas y los sueños que los y las estudiantes tienen en sus planes. Dentro de lo que cabe destacar, se menciona terminar el colegio para estudiar la profesión de su agrado, dentro de las cuales se señalan: médico(a) general, bombero, patóloga, veterinaria, doctora, administrador(a) de empresas, maestra, arquitecto(a), ingeniero(a), arqueólogo(a) y secretaria.

Además, expresan su gusto por realizar diferentes actividades dentro de la institución, por ejemplo, mencionan el equipo de fútbol, los bailes típicos y los talleres que se realizan. Les agrada el centro educativo por su seguridad, buenas instalaciones, limpieza y el silencio en los pasillos. Sin embargo, Pacheco y D’Antoni (2003), al referirse a las instituciones educativas de secundaria, expresan que:

El colegio, como institución, se ubica en un panorama de impresionante soledad, al frente del adolescente: empobrecido en cuanto a recursos, apoyos y confianza, a pesar de representar el único y último baluarte entre el adolescente que sale de su casa y la insegura calle de la Costa Rica de hoy. La sensación es la de que el colegio no ha sabido asumir el desafío para cambiar, aprovechando la creatividad y la fuerza naciente de sus adolescentes. Por el contrario, se identifica con el poder, se esconde, y también los quiere esconder a ellos detrás de uniformes, jerarquías y reglamentos; quiere al frente un mar de caras obedientes y anónimas, sin adornos ni maquillajes que quieran expresar su individualidad, para, cuando menos, identificar a uno de ellos, en la colorida tribu de los jóvenes. La escuela no sabe más que devolver a la casa las infracciones y sus portadores, ignorando el eventual intento de comunicación presente en la infracción misma. (pp. 40-41)

Según Torres (2006), los y las estudiantes de sétimo año necesitan de instituciones que les apoyen en su transición de la primaria a la secundaria, instituciones que les permitan crecer y desarrollarse en todos los ámbitos de su ser integral, que les permitan ser cogestores de su educación. Como se señaló en las consideraciones metodológicas de la investigación realizada, las instituciones que participaron de la investigación poseían algún proyecto en pro de la permanencia y la promoción de los estudiantes. Ésta puede ser una de las razones por las que los estudiantes que participaron en el taller, tengan un concepto optimista de su colegio.

\section{Análisis del Taller de docentes de sétimo año}

A partir de una adaptación, de una propuesta de Guevara (2004), se aplica la técnica Soy docente de sétimo año para trabajar con esta población meta. En grupos, los docentes reflexionan sobre su rol como docentes de sétimo. Posteriormente, comunican, en una plenaria, los resultados. La reflexión se genera a partir de varias preguntas y ejes generadores. Para efectos de este trabajo, se presentan los resultados de una de las preguntas generadoras: ¿Cuál es la importancia de mi labor docente de sétimo año?

Esta reflexión sobre la práctica pedagógica que hicieron los docentes en este taller, "es fundamental y la toma de conciencia acerca de lo que se hace, permitirá transformar esos 'habitus'; 
los cuales están constituidos de estratos sucesivos de esquemas, entre los cuales los más recientes inhiben, de forma voluntarista al principio, luego de forma menos consciente, la puesta en práctica de esquemas anteriores" (Paquay, Altet, Charlier y Perrenoud, 2005, p. 287). A continuación, se resume lo expresado por los y las participantes.

\section{Formación del estudiante en el área académica}

La importancia de la labor de las y los docentes de sétimo año considera la formación del estudiante desde el punto de vista académico, como el aspecto fundamental y de mayor responsabilidad de su labor, de ahí que es el docente quien permite sentar las bases de conocimientos para el estudiante y guiarlos para los próximos años de la secundaria, de tal manera, que se motive y se identifique con cada disciplina específica de este nivel educativo que impactará, de manera positiva, en la futura escogencia de formación profesional en el nivel superior de la educación. En consecuencia, el ejercicio efectivo de esta responsabilidad se relaciona con la posibilidad de que el estudiante logre permanecer en el sistema educativo.

El ejercicio docente es una labor de mediación en la que la facilitación de conocimientos, por parte del educador, es indispensable para que el estudiante desarrolle las habilidades necesarias para tener éxito en el proceso de aprendizaje. Monereo, Castelló, Clariana, Palma y Pérez (1997) establecen la importancia de que en este proceso de mediación el profesional docente, además de ser conocedor de su asignatura, debe ser capaz de reflexionar sobre su didáctica y tomar decisiones oportunas sobre su planeamiento, de manera que ofrezca al estudiante una guía de cómo utilizar, de manera estratégica, los procedimientos de aprendizaje. De esta forma, un mediador competente en términos de capacidad:

1. Parte de los conocimientos previos de sus estudiantes, y es sensible a sus dificultades para hacer explícitos sus procesos mentales.

2. Identifica, en cada momento de bloqueo, el tipo de ayuda que contribuye a desatascar el pensamiento.

3. Espera el tiempo necesario y no se adelanta a la respuesta del estudiantado.

4. Está atento a las iniciativas del estudiantado, así como a los aprendizajes y a las explicaciones erróneas que generan.

5. Desafía lo suficiente mediante las preguntas generadoras, que hacen a los estudiantes reflexionar sobre su proceso de adquisición del conocimiento.

\section{Formación del estudiante en el área personal}

Las y los docentes de sétimo año continúan un proceso de formación del estudiante como persona, el cual considera aspectos que tienen que ver con lo emocional, lo social, lo cognitivo, lo afectivo y los valores que repercuten para toda su vida.

La esperanza de contribuir en esta formación de la persona hace que el docente se considere un hacedor del bien, porque apuesta a la transformación del estudiante en personas críticas, reflexivas, analíticas, con valores como la responsabilidad, la puntualidad, la disciplina y la solidaridad que les permitirán afianzar las herramientas necesarias para enfrentarse a un mundo cambiante y desafiante. 
La consecución de esta meta, requiere de una práctica pedagógica sustentada en una labor de formar con cariño y tacto, pero con rigor en la que se respetan las personalidades y las características de quienes enseñan y de quienes aprenden, así como incorporar las concepciones de amigabilidad, consejería y guía, en sustitución del rol impositivo en el ejercicio de la docencia.

Al respecto, Gavilán y D’Onofrio (2003) establecen que

la propuesta metodológica para responder a esta época, toma como referente la realidad donde el aula debe ser un espacio para la comunicación y el trabajo con otros, intensamente vivido, abierto al mundo circundante, conformado por grupos con objetivos comunes, capaces de transferir a la realidad los saberes construidos. (p. 13)

\section{Transición de primaria a secundaria}

El sétimo año es un nivel educativo en el que se da la transición de primaria a secundaria en el que se aproximan cambios físicos, cognitivos, emocionales y culturales de la población estudiantil, en general.

Es un nivel escolar que logra unir la escuela y el colegio como etapas de un proceso de formación en el que se exige, de parte de los docentes, mucha comprensión, orientación y apoyo para asimilar el cambio para con sus estudiantes; por ello, se hace necesario mantener y consolidar la motivación que traen los educandos de la escuela, para no afectar este proceso de adaptación.

Las y los docentes de sétimo año deben lidiar, al inicio del curso lectivo, con estudiantes que presentan carencias en cuanto a hábitos de estudio, apoyo familiar, organización del tiempo y del espacio, participación activa en el desarrollo de las lecciones y acatamiento de instrucciones, entre otros aspectos, por lo que se requiere una gran dosis de paciencia, compromiso y disposición del docente para encauzar y motivar a sus educandos a incorporar las prácticas que corresponda y superar los retos que algunas representan.

De lo anterior, se consideran muy importantes las visitas previas a cada curso lectivo que puedan llevar a cabo los colegios a las escuelas y a los niños de sexto grado, y que realicen actividades inductivas que los prepare para el conjunto de cambios, con lo que se favorece y estimula la transición de primaria a secundaria (Alfaro et al., 2008b).

\section{Reflexiones finales sobre el tema}

La población de estudiantes de sétimo año presenta características propias, de acuerdo con el desarrollo biopsicosocial y con el estadio maduracional que tienen en el tránsito niñez-preadolescencia-adolescencia.

A continuación, se da cuenta de algunas de las características de la población estudiantil captadas como resultado de las técnicas de recopilación y análisis informativo aplicadas en la investigación.

1. Son personas con posibilidades de diversificar su atención en múltiples intereses que les demandan energía.

2. Pueden realizar varias actividades simultáneamente. 
3. Son audaces, desafiantes, sinceras y sinceros, y manifiestan, con más claridad y directamente, sus gustos y disgustos, sus pareceres y sus rechazos.

4. No conciben la quietud y el silencio grupal. Necesitan estar en contacto con sus pares y compartir con ellos y ellas lo que sienten y piensan; especialmente, en torno a temas de su interés, y, a menudo, no relacionados con lo que se enseña en clase.

5. La autoimagen juega un papel fundamental para ellos y ellas, por lo que es común pasarse fotografías, mirarse al espejo, sacarse las cejas, peinarse, perfumarse, cuchichear temas de su interés. Ambos sexos se interesan por su apariencia, y llevan consigo cosméticos y perfumería.

6. Las tareas y/o los trabajos asignados por los y las docentes no siempre son de interés para ellos y ellas; por lo que requieren motivación, ya que si no son significativos, tienden a incumplirlos.

7. En ausencia de liderazgo o estimulación para la realización de las actividades de aprendizaje, se autodesorganizan y se enfocan a satisfacer sus variados gustos, pues sienten especial atención por todo lo nuevo, desconocido o famoso, y pueden desatender las indicaciones y las tareas.

8. Les despiertan más interés las asignaturas y las temáticas vinculadas con la vida cotidiana y las que no los estimula a ausentarse, ser impuntuales, interrumpir o abandonar, constantemente, la clase.

9. Se agrupan por afinidad, generalmente, entre los del mismo sexo.

10. No muestran un gran interés por las calificaciones y los promedios obtenidos; lo que los motiva es ganar puntos para su calificación del período lectivo, como un logro inmediato, enfatizando en el aquí y en el ahora, sin tener claras sus metas y su proyecto de vida. Perciben el colegio como una obligación impuesta por sus padres, y como un puente para llegar a la universidad.

11. Se limitan a responder lo que pregunta el profesor o la profesora, y realizan pocas consultas sobre la temática en estudio.

12. El aspecto académico no es esencial en sus autovaloraciones y, generalmente, no han proyectado sus aspiraciones aún; muchas veces, ni prevén las consecuencias de sus acciones.

13. Son entusiastas y tienen mucha vitalidad. Muestran mucha expresividad y desinhibición.

14. No sienten compromiso conceptual ni paradigmático, por lo que expresan más flexibilidad y apertura a nuevos aprendizajes y a la adquisición de hábitos y destrezas, y a rectificarlos y modificarlos cuantas veces lo requieran.

15. Son parte de la globalización creciente, son más presente y futuro que sus educadores, porque tienen menos que desaprender de un pasado que apenas conocen, por lo que son más adaptables.

\section{Referencias}

Alfaro, M.; Brenes, A.; Gamboa, A.; Jiménez, S.; Martín, J.; Ramírez, A.; Vargas M. \& Zúñiga, A. (2008a). Construcción del perfil de desempeño docente para el sétimo año de la educación pública. Informe de Investigación. Heredia, Costa Rica. Publicación sin sello editorial para el Centro de Investigación y Docencia en Educación de la Universidad Nacional. 
Alfaro, M.; Gamboa, A.; Jiménez, S.; Martín, J.; Ramírez, A. \& Vargas, M. (2008b). Construcción del perfil profesional docente de sétimo año: respuesta a una necesidad actual. Revista Educare, 12 (2), 32-45.

Alfaro, M.; Gamboa, A.; Jiménez, S.; Martín, J.; Ramírez, A. \& Vargas, M. (2009). Diversidad estudiantil en el sétimo año de la educación secundaria pública costarricense. Revista Educare, 13 (1), 27-39.

Castro, M.; Díaz, M.; Fonseca, H.; León, A.T.; Moraga, L.; Ruíz, S. \& Umaña, W. (2007) Factores de éxito en la transición a sétimo año. (Informe Final de investigación del Proyecto Perfiles, dinámicas y desafíos de la educación costarricense, Etapa II): Heredia, Costa Rica: Centro de Investigación y Docencia en Educación, Universidad Nacional.

García, F. Portillo, J. Romo, J. \& Benito, M. (2007). "Nativos digitales y modelos de aprendizaje". Actas IV Simposio Pluridisciplinar sobre Diseño, Evaluación, y Desarrollo de Contenidos Educativos Reutilizables, Bilbao. Recuperado el 25 de enero de 2008, de http://spdece07.ehu. es/actas/Garcia.pdf

Gastón, A. (1996). Interacción social y desarrollo. En Lecturas sobre el desarrollo de la inteligencia y del pensamiento. Serie de Publicaciones $\mathrm{N}^{\circ}$ 1. San José, Costa Rica: Publicaciones del SIMED.

Gavilán, M. \& D’Onofrio, S. (2003). Re-pensar al adolescente de hoy y re-crear la escuela. Recuperado el 5 de abril de 2008, de http://www.educared.org.ar/vicaria/adjuntos/tema-mes/ Conf-Adolescencia.pdf

Guevara, R. (2004). Material fotocopiado del Departamento de Orientación y Vida Estudiantil. Ministerio de Educación Pública de Costa Rica.

Guitar, R. (2002). Las actitudes en el centro escolar. Reflexiones y propuestas. Barcelona, España: Editorial GRAÓ.

Labarrere, A. (2008). Bases conceptuales de la mediación y su importancia actual en la práctica pedagógica. Summa Psicológica UST, Vol. 5, № 2, 87-96.

Monereo, C., Castelló, M., Clariana, M., Palma, M. \& Pérez, M. (1997). Estrategias de enseñanza y aprendizaje: formación del profesorado y aplicación en la escuela (4 ed). Barcelona, España: Editorial GRAÓ.

Obiols, G. \& Di Segni, S. (1995). Adolescencia, Posmodernidad y Escuela Secundaria. La crisis de la enseñanza media. Buenos Aires, Argentina: Kapelusz Editora.

Pacheco, X. \& D’Antoni, M. (2003). ¿Por qué?: Jóvenes ante el desorden mundial. Heredia, Costa Rica: Educología, Universidad Nacional. 
Paquay, L., Altet, M., Charlier, E. \& Perrenoud, P. (2005). La formación profesional del maestro. México D. F.: Fondo de Cultura Económica.

Prensky, M. (2001). Digital Natives Digital Immigrants. Recuperado el 5 de abril de 2008, de: http://www.marcprensky.com/writing/Prensky\%20\%20Digital\%20Natives,\%20Digital\%20 Immigrants\%20-\%20Part1.pdf

Rojas, Y. (2007). La formación de educadores desde la propuesta del CONARE: Hacia un modelo educativo para mejorar la calidad de la educación costarricense. Congreso Pedagógico Nacional: Formación profesional del educador y la educadora costarricenses. San José, Costa Rica: Autor.

Torres, R. (2006). Los nuevos paradigmas en la actual revolución científica y tecnológica. San José, Costa Rica: EUNED. 